\title{
PHYSICS OF HOT HADRONIC MATTER AND QUARK-GLUON PLASMA
}

\author{
E.V. Shuryak \\ Physics Department \\ Brookhaven National Laboratory \\ Upton, New York 11973
}

\section{Received by OSTI}

AUG 201990

\begin{abstract}
This Introductory talk contains a brief review of the current status of theoretical and experimental activities related to physics of superdense matter. In particular, we discuss latest lattice results on the phase transition, recent progress in chiral symmetry physics based on the theory of interacting instantons, news in the theory of QGP and of hot hadronic matter, mean $p_{t}$ and collective flow, the shape of $p_{t}$ distribution, strangeness production, $J / \psi$ suppression and $\phi$ enhancement, two puzzles connected with soft pion and soft photon enhancements, and some other "ultrasoft" phenomena.
\end{abstract}

Invited talk presented at

Quark Matter 90

Menton, France, 7-11 May 1990

This manuscript has been authored under contract number DE-AC02-76CH00016 with the U.S. Department of Energy. Accordingly, the U.S. Government retains a non-exclusive, royalty-free license to publish or reproduce the published form of this contribution, or aliow others to do so, for U.S. Government purposes.

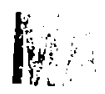




\title{
PHYSICS OF HOT HADRONIC MATTER AND QUARK-GLUON PLASMA
}

\author{
E.V. Shuryak
}

Physics Department

Brookhaven National Laboratory

Upton, New York 11973

This Introductory talk contains a brief review of the current status of theoretical and experimental activities related to physics of superdense matter. In particular, we discuss latest lattice results on the phase transition, recent progress in chiral symmetry physics based on the theory of interacting instantons, news in the theory of QGP and of hot hadronic matter, mean $p_{t}$ and collective flow, the shape of $p_{t}$ distribution, strangeness production, $J / \psi$ suppression and $\phi$ enhancement, two puzzles connected with soft pion and soft photon enhancements, and some other "ultrasoft" phenomena.

\section{INTRODUCTION}

Starting this Introductory talk at Menton, at the 8-th of the "Quark Matter" conferences, I probably should not go into discussions of the general reasons for studies of high multiplicity hadronic systems produced in high energy collisions. Let me only mention, that the main inspiration of this activity came from $\mathrm{QCD}$ and is related to possible manifestation of the phase transition, in which the QCD vacuum is "melted" and new phase of matter, the so called quark-gluon plasma (QGP) ${ }^{1}$ is produced. Let me also refer to previous conferences ${ }^{2}$, where the first experimental data concerning high energy nuclear collisions at CERN and Brookhaven were discussed. I hope to make a brief review of the whole field, keeping to news which appeared since the previous conference. The list of subjects is given in the Abstract above. There is no "summary" or "discussion" at the end: the entire text is of this type

\section{RECENT NEWS ABOUT PHASE TRANSITIONS FROM}

\section{LATTICE CALCULATIONS}

Let me start this section with the comment, that lattice "numerical experiments" are rapidly reaching the level of "normal" high energy experiments, in terms of big collaborations, cost of the projects, etc. There have also been significant ideological changes during the last couple of years: the failure of the previously very popular "quenched approximation"

This manuscript has been authored under contract number DE-AC02-76CH00016 with the U.S. Department of Energy. 
(based on idess of large $N_{c}$ limit), according to which light quarks in the QCD vacuum, are but an unimportant complication. However, one cannot really extrapolate from $N_{f}=0$ point to QCD. Moreover, even the valuc of the strange quark mass seems to be important, for $N_{f}=2$ and 3 cases are quite different. There are radical changes of the vacuum structure as quark masses become less than 50-100 Mev, e.g., new first order phase transition is found there. Another important fact is strong dependence of $T_{c}$ on the number of flavours: from $N_{f}=0$ to 4 the ratio $T_{c} / m_{\rho}$ significantly decreases.

Not being able to cover these results in details (see review by B. Petersson in this proceedings), I concentrate on one general physical question, which, I hope, is more or less clarified. In the QCD vacuum we have two non-perturbative phenomena, confinement and chiral symmetry breaking. Are they related or not? Or, in a more definite way, are there two independent phase transitions ${ }^{3}$ on the phase diagram with different physics, or just one?
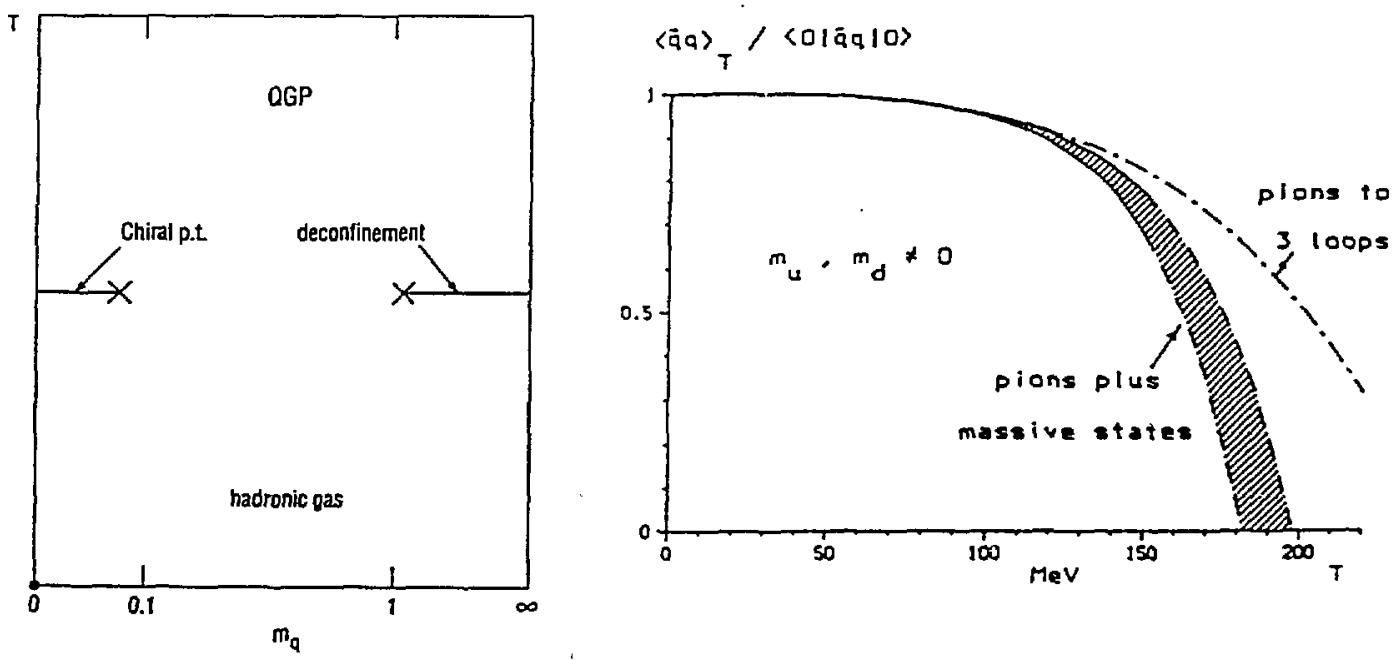

Figure 1: (a) Schematic phase diagram of hadronic matter for $N_{f}>2$ on the plot quark mass $m$ versus temperature. (b) Quark condensate versus temperature according to ${ }^{8}$.

Numerical studies done during the last few years have shown that the former alternative seems to be case. The resulting picture can be described as follows. Let all our $N_{f}$ quarks have the same mass $m$, so we may plot simple phase diagram on the temperature-mass plane, see Fig. 1(a). Roughly speaking, there are three different worlds, with completely different features of the phase transition. The "glwonic world" at the right has only quarks with $m>1 \mathrm{GeV}$, as if all quarks are charmed. It was shown (see recent discussion in Ref. 4) to have strong first order deconfinement transition. Another extreme is the world of light quarks. Here there appears to be another first order transition, connected with chiral symmetry restoration ${ }^{5}$. It is seen for quark masses below $50-100 \mathrm{MeV}$ for $N_{f}>2$, while for 
$N_{f}=2$ transition is probably only second order. If two masses are light and $m_{s}$ is changed, the first order signal disappears somewhere around $100 \mathrm{MeV}$.

In the middle of Fig. 1(a) there is an "intermediate mass world", which attracted the most attention and computer power during the last year, especially of "homemade" 16 Gigaflop 256 processor complex ${ }^{6}$ at Columbia. Since no first order transition is seen here, it is demonstrated that deconfinement and chiral restoration transitions are separated at the phase diagram. Respectively, the old idea that they are indeed two different phenomena has obtained new support. Real QCD happens to be close to the end of the chiral transition zone. But Columbia data seems to indicate the absence of first order transition.

\section{PROGRESS IN UNDERSTANDING OF CHIRAL SYMMETRY RESTORATION}

The classical papers of the early '60's like Ref. 7, considered pion and sigma fields and explained in their terms how the latter may obtain non-zero expectation value and how the former becomes massless. This approach can also explain chiral symmetry restoration at high temperatures. In connection to that, let me show Fig. 1(b) from a recent paper ${ }^{8}$, where "melting" on the quark condensate was calculated in chiral perturbation theory. These results are very general and hold for small $T \ll T_{c}$.

However, real undersianding of chiral symmetry restoration can only be reached if we consider the problem at a more fundamental level, starting from quarks and gluons. Chiral symmetry is a consequence of the fact that in massless theory with vector interaction right and left-handed quark fields are completely independent, so independent rotations in flavor space can be made. The quark mass $m$ plays, in this problem, the same role as infinitely small magnetic field in the iron magnetization problem: it breaks the symmetry. The question is whether it produces $100 \%$ asymmetric vacuum, even for infinitely small $\mathrm{m}$.

Formally, the so-called quark condensate can be connected with the eigenvalue spectrum of the Dirac operator $\widehat{D}=\gamma_{\mu}\left(i \partial_{\mu}+g A_{\mu}^{a}(x) t^{a}\right)$ defined as usual $\widehat{D} \psi_{\lambda}(x)=\lambda \psi_{\lambda}(x)$. There exists the following general expression $\langle\bar{\psi} \psi\rangle=\sum_{\lambda} m /\left(\lambda^{2}+m^{2}\right)$ which has nontrivial limit $m \rightarrow 0$ if there exist eigenvalues $\lambda$ arbitrary close to zero. (Unfortunately, it is exactly these small eigenvalues which are very difficult to get on the lattice.)

Now, what is the physical nature of these quark states with small "virtuality" $\lambda$ ? There is good evidence that one can understand them without real evaluation of all eigenvalues for any gauge field configurations, as lattice people try to do, for they are generated by specific type of gauge field configurations, the instantons. Let me remind that they describe tunneling between topologically different gauge field vacua and that such iunneling changes occupations of fermionic states. Therefore, each instanton can be considered ${ }^{9}$ as the vertex, producing a quark-antiquark pair of each light flavor.

Using this language, one may say that the $Q C D$ vacuum is a complicated matter made of instantons, connected by quark lines ${ }^{10}$. The question is, what is the structure of this matter. If it is a kind of a "crystal", we get spontaneous breaking of translational and color symmetries. If it is a "polymer" or "liquid", one has breaking of cliral symmetry. Finally, 
if it is a "gas" made of instanton-anti-instanton molecules, it has no small $\lambda$ states and therefore chiral symmetry is unbroken.

These qualitative ideas I have already discussed at the previous conference in Lennox, and since then changes are really radical: instead of phenomenological "instantcn liquid model" 10 , and simple mean field approximations ${ }^{11,12}$, we have today a developed quantitative theory ${ }^{13}$, including 't Hooft interaction in all orders.

Results of these calculations are in remarkable agreement to what we observe in the real world. The QCD vacuum was indeed shown to be the "instanton liquid", breaking the chiral symmetry and producing quark condensate of the needed magnitude ${ }^{14}$. At $T>200 \mathrm{MeV}$ chiral symmetry restoration is observed as a rapid transition into molecular phase. This theory provides a natural explanation of the fact that for $m>50-100 \mathrm{MeV}$ ihis transition disappears: instantons in this case form "atomic gas". This low mass scale comes from typical $\lambda \sim \rho^{2} / R^{3}$, where $\rho, R$ are typical instanton size and separation.

Moreover, calculation of the mesonic correlation functions ${ }^{15}$ with different quantum numbers show very non-trivial behaviour, and all the details (sometimes with surprising accuracy) agree with experiment. Certainly I cannot discuss them here, but in many respects this theory works much better, than the lattice one.

\section{NEWS IN THE THEORY OF QUARK-GLUON PLASMA}

Studies of QGP can be divided into three categories, related to typical momenta of constituents: (i) "physics of typical quarks and gluons", with momenta of the order $T$; and two sectors of "soft physics", with momenta of the order of (ii) $O(g T)$ and (iii) $O\left(g^{2} T\right)$.

The first direction is mainly related with perturbative calculations of gluonic radiative corrections to elementary processes. As an example, let me refer to recent papers ${ }^{16}$ on gluonic corrections to dilepton production from QGP.

The progress in category (ii) is mainly methodical. Interesting gauge-independent statements about multi-leg Green functions outside the mass shell (!) were discovered ${ }^{18}$. Another important news: after different answers for the gluon damping rate in QGP, suggested earlier, now in ${ }^{17}$ it is found that $\gamma_{\text {plasmon }}=\gamma_{\text {transverse }}=+6.635\left(\alpha_{s} T / 4\right)$, where the coefficient is positive and gauge-independent. Looking at these works from the practical side, let me mention the recent calculation of low mass dilepton production ${ }^{19}$, in which significant "vertex corrections" are found. In usual language, apart from traditional quark annihilation, there is also bremsstrahlung related to quark-gluon scatterings. New suggested phenomena ${ }^{20,19}$ are peaks in the dilepton mass spectrum related to specific resonances with "modified quarks" in QGP. Unfortunately, observability of all these phenomena is problematic, for soft dileptons from QGP are buried under much larger radiation from hadronic phase and usual background coming from hadronic decays.

Questions of the third category have probably even less practical importance at the moment, but are instead very interesting for theoretical reasons. Due to the absence of magnétic screening ${ }^{1}$, at momenta $O\left(g^{2} T\right)$ hot QCD becomes really nonperturbative, with 
power infrared divergencies. At Lennox, J. Polonyi discussed a possible solution of this problem: breakdown of color symmetry and appearance of a kind of Higgs phase, with $\left\langle A_{0}^{a}\right\rangle \neq 0$. I do not think we really have new convincing arguments for that. K. Kajantie, who recently made lattice studies of the problem, will tell us more about it.

\section{NEWS ABOUT HOT PION MATTER}

Whether or not QGP is produced in high energy nuclear collisions, they certainly produce hundreds (and, with lead beam, it will be thousands) of pions. Interferometry tells us that resulting hadronic systems reach radii 6-10 $\mathrm{fm}$ at about nuclear density, so they become much larger than even the heaviest nuciej. Certainly properties of hadronic matter can, and should be understood much better, using these data.

Theory of hot hadronic gas has radically changed during the reported period. First of all, now we have much more detailed understanding of its thermodynamics and kinetics. Second, it is realized that pions (and perhaps nucleons) in hot hadronic matter are good "quasiparticles". All that, taken together ${ }^{35}$ shows that this matter, far from being an ideal pion gas, is rather a liquid, in which strong attractive interaction among secondaries plays an essential role. Like in ordinary liquid, particles have some difficulties on the boundary to leave the system, and this makes important observable effects.
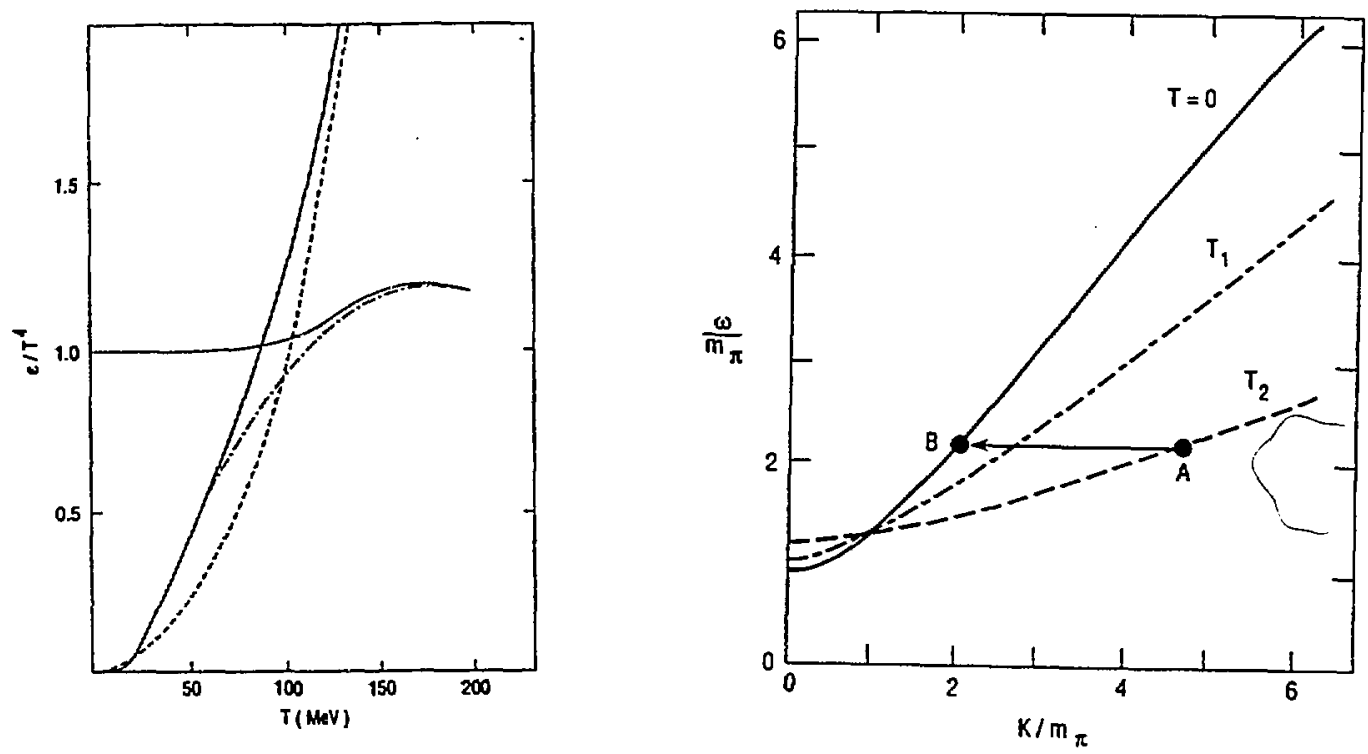

Figure 2: Ratio of the correlation functions of currents with quantum numbers of corresponding mesons to those, corresponding to free quark propagation, versus the distance $x$ between the currents. The points are from the instanton liquid calculation ${ }^{15}$. 
Let us first remind some general facts about the pions. For massless quarks they should also be massless, exactly for the same reason as e.g. acoustical phonons: both are Goldstone modes, the remnants of spontaneously broken symmetry. At small $T$ the pion gas is dilute, and ideal gas approximation is justified. At larger temperatures interaction become significant. Let me emphasize that, due to their Goldstone nature, interaction of pions is also quite specific. In the chiral world, pions with $k \rightarrow 0$ cannot interact at all. (Exactly due to this fact, acoustical phonons propagate distances much larger than molecule free paths, thus we can hear each other.) This feature makes this gas even more ideal at small $T$.

Accounting for momentum-dependent pion-pion interaction, one can use chiral perturbation theory if $T \ll F_{\pi}$, otherwise one has to include resonances more accurately. Recent results $^{21}$ on energy density are shown in Fig. 2(a). For the ideal massless gas this ratio is close to one, and interaction of pions makes some modifications (see the dotted line). Much more dramatic modifications are due to non-zero pion nuass and hadronic resonances (the solid line). We also show simple parameterization for the "pion+resonances" suggested by the author many years ago ${ }^{22}: \epsilon(T)=T^{5} / T_{0}^{2}, T_{0}=100 \mathrm{MeV}$ which is shown by the dashed line. One can see that it approximately describes the energy density dependence on temperature over the whole interval. Similar results were also recently obtained in Ref. 23 .

Now we discuss kinetics of the pion matter. Again Goldstone nature of the pion plays an important role: apart from small effects related with scattering lengths, the $\pi \pi$ cross section is essentially proportional to pion momenta. Therefore mean time between collisions depend on $T$ as follows ${ }^{24}: \tau_{\text {coll }}=$ const $F_{x}^{4} / T^{5}$ Goity and Leutwyler have recently made extensive calculations ${ }^{25}$ supporting this expression and getting more accurate const $=12$. Note, that at $T=200 \mathrm{MeV} \tau_{\text {coll }}$ is small, $.7 \mathrm{fm} / \mathrm{c}$, but at our other extreme $T=100 \mathrm{MeV}$ it is already about $25 \mathrm{fm} / \mathrm{c}$, comparable to the lifetime of even the largest fireballs considered. Another useful way to look at these results is to consider the ratio of imaginary to real parts of the pion energy: $\operatorname{Im} E_{\pi} / \operatorname{Re} E_{\pi}=.03\left(T / F_{\pi}\right)^{4}$ It is still only about .1 at $T=140 \mathrm{MeV}$, but become of the order 1 at $T_{c}=200 \mathrm{MeV}$. Therefore, in the former case the pions are still propagating rather well, while in the latter case they are quickly absorbed.

Now we come to the central point of this section: pions are not only scattered in hot matter, but they interact with many particles simultaneously and change their properties. The main pion-pion interaction is proportional to pion momenta, respectively our collective potential grows with momentum as well. Such a situation is common for all Goldstone modes, let us mention two well-known examples of cases in which the dispersion curve is strongly modified at larger $k$, so that even secondary minimum is developed. (i) The famous dispersion curve of elementary excitations in liquid $\mathrm{He}^{4}$, measured by neutron scattering experiments. The secondary minimum of this curve was suggested by Landau and called the "roton" minimum. (ii) Pion dispersion curve in cold dense nuclear matter. With increasing nuclear density, modification of pions may become so strong that the secondary minimum may reach zero (at some critical momentum $k_{0}$ ), leading to vacuum rearrangement, or Migdal pion condensation. 
The expected qualitative behaviour of the dispersion curve in hot pion gas is shown in Fig. 2(b) : the interaction is slightly repulsive at small momenta but becomes attractive at larger ones. We do not want to have many free parameters, so we use simple parametrisation $\omega(k)=\left(m_{\pi}^{2}+u(T)^{2} k^{2}\right)^{1 / 2}$, introducing the temperature-dependent slope parameter $u(T)$. Its temperature dependence can be estimated in the same way as Landau did for quasiparticles in liquid $H e^{4}$. We know that $\epsilon(T)$ grows faster than that for the free pions, so let us assume that it can be represented by a gas of modified "quasipions". The resulting $u(T)$ is close to one up to $T=150 \mathrm{MeV}$, and then decreases, up to about $1 / 2$ at $T_{c}$.

\section{OTHER HADRONS AT NON-ZERO TEMPERATURES, $\phi$-RELATED PHYSICS}

In first approximation, one may start with the naive constituent quark model, and say that roughly speaking mesonic and baryonic masses are nothing else but 2 or 3 "constituent quark masses". As the latter are also related to chiral symmetry breaking; one may expect that at $T=T_{c}$ all masses of hadrons turn to zero. The simplest scenario ${ }^{26}$ is that all mass scales are the same, therefore $m_{\text {hadrons }}(T) \sim f_{\pi}(T) \sim \mid\left\langle\bar{\psi} \psi>\left.(T)\right|^{1 / 3} \sim\left(1-T^{2} / T_{c}^{2}\right)^{1 / 6}\right.$ : (the last equality comes e.g. from Fig. 1(b)).

In second approximation we discuss why all mesons are not alike. This is essentially due to instanton-induced interaction between quarks and anti-quarks. For example, $\eta^{\prime}$ is so heavy because it is repulsive, while the pion is so light because it is attractive. Asking what happens to these effects at higher $T$, one get a simple answer: instantons are suppressed ${ }^{1}$ at high $T$. Let me speculate, that phenomena, which are first order in the instanton density, should disappear earlier than effective mass (proportional to its smaller power). If so, then first all meson masses come together, and only later they all come down. In particular, $\eta^{\prime}$ mass is expected to decrease faster than that of "normal" mesons like $\rho, \omega, \phi$. For pion we have competition of two trends, and chiral perturbation theory suggests a slight increase ${ }^{8}$ at small $T m_{\pi}(T) / m_{\pi}(0)=1+T^{2} / 48 F_{\pi}^{2}+\ldots$.

But how one can observe all that? I think the best case is the $\phi$ meson. There are several reasons for that. (i) $\phi$ lifetime is about $45 \mathrm{fm} / \mathrm{c}$, which is comparable to the expected lifetime of hadronic fireball in heavy ion collisions. It means that (unlike $J / \psi$ ) there should be significant fraction of $\phi$ decaying inside the matter. (ii) The $\phi$ mass is just a bit above $2 m_{K}$. Therefore, its decays are very sensitive to relative shifts of dispersion curves of $\phi$ and $K$, (this point was also independently made by $R$. Pisarski) (iii) $\phi$ has isospin zero, and pion scattering at low energies is proportional to isospin. So we expect $\phi$ to be modified less than $K$. If $K$ mass goes up or down a little, the main decay channel of $\phi$ is blocked or enhanced. (iv) Two modes of $\phi$ decay, $K^{+} K^{-}$and $e^{+} e^{-}$ones, are measurable, so one can directly check whether the branching ratio is different from that in vacuum. (This point was suggested by D. Lissauer in discussion.). (v) With good enough resolution, one may also look for the changes of the $\phi$ peak shape in invariant dilepton mass.

Other resonances, e.g. $\rho, \omega, \eta, \eta^{\prime}$, are also interesting although only $e^{+} e^{-}$and $\gamma \gamma$ models are observable. Recent studies of the nucleon modification ${ }^{55}$ showed, that the nucleon mass 
is shifted only by about $10 \mathrm{MeV}$ up at $T=150 \mathrm{MeV}$, while its imaginary part at this $T$ is already about $100 \mathrm{MeV}$.

\section{NUCLEAR STOPPING, THERMALIZATION AND ALL THAT}

I will be very brief considering this topic, making only few critical remarks related to the following general questions. Can we understand data on nuclear collisions using those for the $p p$ and the $p A$ ones? Should we even try to do it?

Before experiments with nuclear beams started, people had used available "event generators" fitted to describe $e^{+} e^{-}$and $p p$ data to describe nuclear collisions. Certainly it was useful to evaluate acceptances etc. These "event generators" indeed can describe $E_{t}$ spectra, roughly give the pion spectra, and even (somewhat worse) the nucleon ones. However, one should not really take them for QCD predictions. Moreover, I think at the moment it is even hopeless to try to create real theory of this complicated phenomenon. Too many difficult questions remain open.

(i) First of all, we know too little about "strings" and their properties. It is also not quite certain how one should attach these strings to quarks and gluons. (ii) We also do not know what happens if many such strings overlap in space. In available CERN experiments their density is few strings per $\mathrm{fm}^{2}$ of the cross section, and few times more in future lead beam experiments. And still some people argue that they do not interact! (iii) Even if we have independent strings, which independently produce hadrons, we certainly have rescatterings before secondaries come out of the system. Therefore, any agreement between the particle spectra obtained in such. "pp-based" models (which ignore "trivial final stage interaction") can be but misleading coincidence.

But should we really try to understand all the details of the dynamics of nuclear collisions? I do not think so. After all, we actually want to learn from nuclear collision experiments very simple things, like equation of state. The whole project started with the idea, that QGP is much simpler than, say, the proton. (For example, one can certainly describe water flow in tubes or its boiling in the pot in terms of equation of state, without any understanding of such details as the scattering amplitudes of $\mathrm{H}_{2} \mathrm{O}$ molecules.) It is production of the macroscopic system and its description in macroscopic terms, which is the ultimate aim of this field.

Experiments do demonstrate rather nice stopping (even at CERN energies) in terms of nucleon energy loss. Pion rapidity distribution is surprisingly close to predictions of Landau hydrodynamics. Does it really mean, thac there is rapid thermalization? This question is still open, for we do not have reliable estimates for the initial thermoplastic time. People use Bjorken $1 \mathrm{fm} / \mathrm{c}$ value as a guess, although both old perturbative estimates of gluonic mean free path ${ }^{29}$ and estimates based on "color rope" model ${ }^{28}$ give something shorter, $1 / 2$ to $1 / 3 \mathrm{fm} / \mathrm{c}$. I do not think theory can now do better: only experiments sensitive to initial stage of the process (especially direct photons and dileptons) can shed light on it. 
8. MEAN $p_{t}$ AND THE COLLECTIVE FLOW

Taking into account uncertainties concerning the origin of the observed rapidity distribution, let us consider transverse momenta. Transverse flow is certainly the most obvious method to measure the equation of state, pressure versus energy density. However, when one approaches the phase transition region, the equation of state becomes very "soft" ${ }^{30,56}(p \ll \epsilon)$ and only small flow velocity is expected. Only when the initial energy density significantly exceeds that needed for QGP formation, collective flow is expected to become noticeable. Calculations of hydro expansion with bag-model type $\operatorname{EOS}^{31}$ gave predictions shown in Fig. 3(a). The existence of some plateau in its middle is the consequence of softness of EOS in the "mixed phase".

Independence of mean $p_{t}$ on $E_{t}$ or multiplicity was indeed observed by CERN experiments. This point is not much discussed, experimentalists usually say that "nothing new is observed here". However, it demonstrates softness of EOS, indicating that we have approached the phase transition domain.
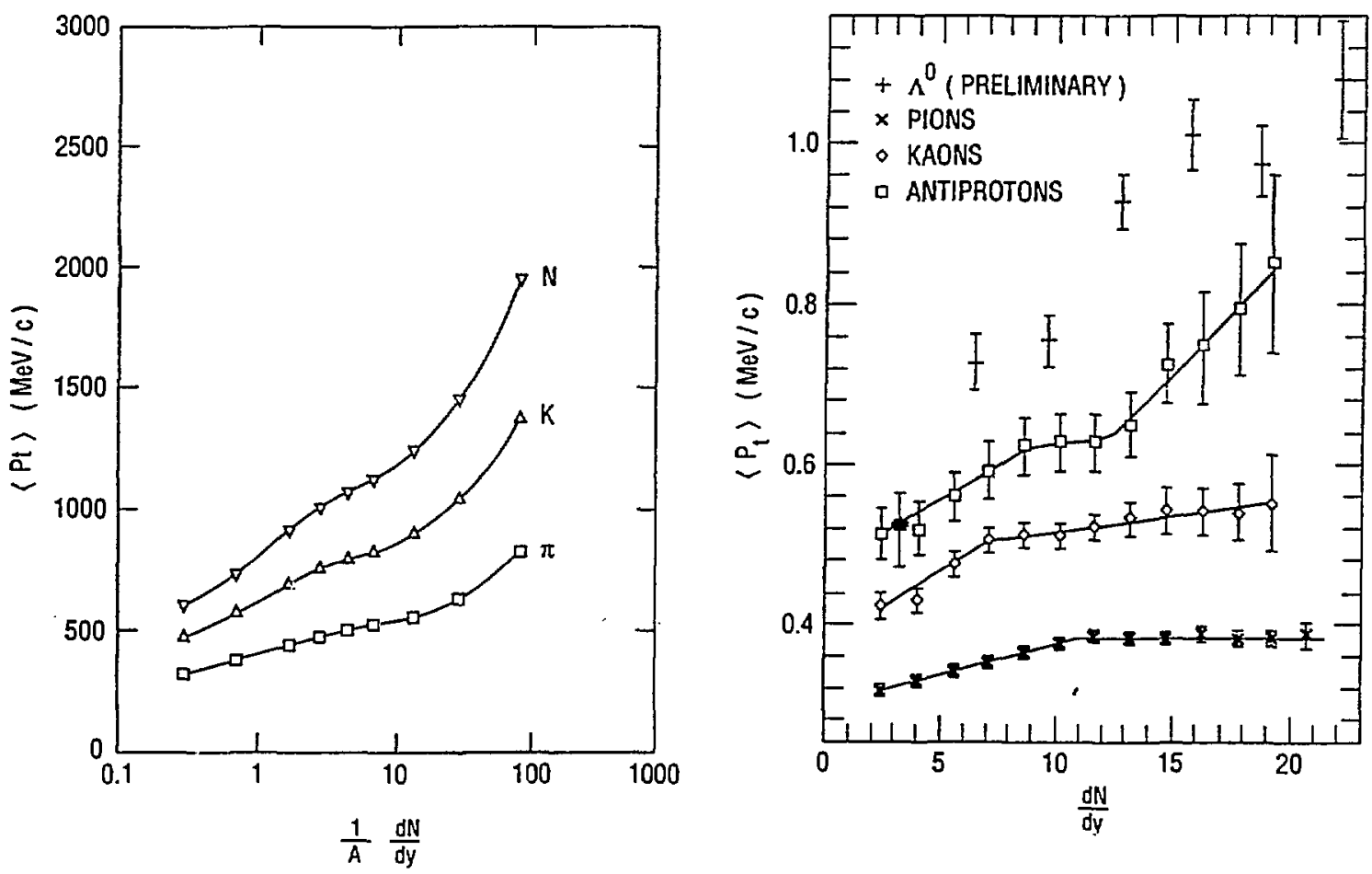

Figure 3: Mean transverse momenta of various secondaries versus $d N / d y$ (effective energy density). (a) Hydrodynamics with phase transition ${ }^{31}$; (b) FNAL data ${ }^{32}$. 
The first data which, I hope, shows some QGP flow are TEVATRON data which was discussed in Lennox. Now this group has data for identified secondaries ${ }^{32}$, see Fig. 3(b). Selecting large multiplicity events in $1.8 \mathrm{TeV} p \bar{p}$ interaction, they have shown that $\left\langle p_{t}\right\rangle$ of heavier secondaries start to grow with multiplicity, if $N_{c} \gtrsim 100$. The shape of the observed dependence is provocatively similar to hydro calculations. These events indeed correspond to very high initial energy density, exceeding those needed for QGP formation. (Note also, that such growth is against the obvious kinematic effects. It is also against the "mini-jet" type models: such jets, as seen in few $\mathrm{GeV} e^{+} e^{-}$annihilation, are very poor in $p, \Lambda$, and their production should rather affect mesonic spectra, not baryonic ones, as observed.)

\section{THE SHAPE OF THE $p_{t}$ SPECTRA}

Roughly speaking, pion spectra observed in nuclear collisions at CERN can be described by two exponents, with slopes about 50 and $200 \mathrm{MeV}$, while other secondaries show only the second component. It is well seen e.g. from NA35 data ${ }^{33}$, Fig. 4(a). Note that it is $m_{t}=\left(m^{2}+p_{t}^{2}\right)^{1 / 2}$ spectra, divided by extra $m_{t}^{1 / 2}$ : then thermal spectra integrated in $p_{t}$ should be exponential. All lines have the same slope, which is $200 \mathrm{MeV}$. This is not the case neither at lower energies (AGS), nor at larger ones (Tevatron), where heavier secondaries have larger "effective temperatures".

Discussing interpretation of this shape, I have to start with that suggested in Ref. 34, much discussed at Lennox. At first glance, the concave shape of the pion $p_{t}$ distribution indeed suggests very low break-up temperature $T_{f}=50-100 \mathrm{MeV}$, while additional hydro motion (of the outer part of the matter) can describe the tail. However, this picture is hardly true because of the following reasons. (i) Such low $T_{f}$ definitely contradicts kinetics of the pion gas: for $T<100 \mathrm{MeV}$ we do not expect any collisions. (ii) Even if some unknown mechanism manages to cool the pion system to such low $T$, the size of the pionic source is then much larger than that measured by interferometry. (iii) Also, the idea of one single break-up temperature is not really valid, for, by definition, near break-up the system size and particle mean free path are comparable. Cascade-type Monte Carlo (e.g. my calculations reported in Ref. 35) show in fact significant contribution of the particles, "evaporated" in the whole interval $T=100-200 \mathrm{MeV}$. (iv) If expansion velocity $v$ is as large as in Ref. 34, there is no time to cool the system. I found reasonable spectra only with $v=.1-.2$.

Let me postpone further discussion of "soft" enhancement, and now discuss another interpretation $r^{*}$ the second component of the spectra. Another useful way to analyze spectra is to represent them as a sum of the thermal ones, convoluted with the so-called "temperature profile function"29 $F(T)$. Assuming that during its expansion the system spends much time near critical temperature (in the mixed phase?), one expects to find a peak in $F(T)$ at $T=T_{c}$. Results of my' attempts to convert available data into $F(T)$ are shown in Fig. 4(b). Some bump can indeed be seen in the $225 \mathrm{MeV}$ bin compared to 

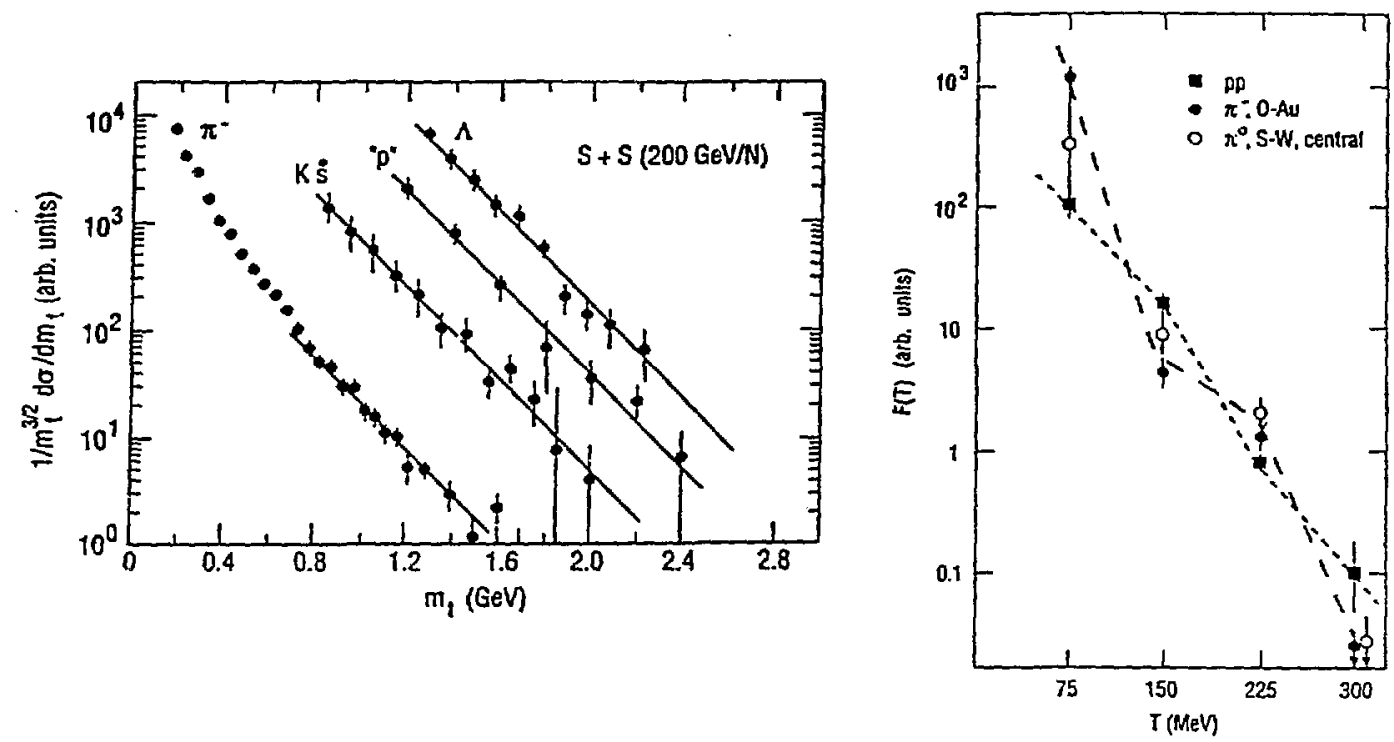

Figure 4: (a) Transverse mass spectra for various types of secondaries for SS collisions, NA35 collaboration. (b) Temperature profile function $F(T)$ versus temperature (for comparison, each spectrum is normalized to one). The open and closed points correspond to WA80 and HELIOS data for reactions indicated, while stars represent $p p$ data from ISR.

its neighbours, or compared to $p p$ data. Obviously, one needs much better data to check whether it is indeed evidence of a peak.

These observations (and also those about composition of secondaries, see strangeness section) make one think about "evaporation" from the mixed phase. If so, this slope $200 \mathrm{MeV}$ is nothing else but the critical temperature $T_{c}$. However, in order to make this interpretation really convincing, one also has to do some other things. One has to show that $T=200 \mathrm{MeV}$ signal is also seen by "penetrating probes". Also, one can check whether flow is indeed small; comparing spectra of different secondaries at small $p_{t}$, where they are still non-relativistic. (All ultrarelativistic particles are similarly affected by the flow).

\section{PRODUCTION OF NEW FLAVORS}

Enhanced production of new types of quarks, charmed and strange ones, in the plasma phase has been repeatedly emphasized starting from the very first works on the subject ${ }^{2}$. It was suggested to be a very nice "thermometer", similar to the one used by doctors, sensitive to the highest temperature of the process. However, this thermometer needs a careful calibration.

Latest studies of strangeness production has shown, that it may be much more intensive in hadronic gas than was estimated previously. The consequence is that global $K$ and $\Lambda$ 
production are not really a good signal for QGP formation, but anti- $\Lambda$ and multistrange baryons are probably sensitive to it. In spite of experimental difficulties with observation of charmed particles, perhaps their leptonic trace should be seriously considered for RHIC and LHC experiments.

Discussing strangeness in more details, let me start with experimental observations at low energies. Significant strangeness enhancem int is seen in antiproton annihilation on nuclei at energies as low as $4 \mathrm{GeV},{ }^{36}$ where particle spectra show in this case temperature as low as $60 \mathrm{MeV}$, or the excitation energy density orders of magnitude smaller than needed for QGP. (Nevertheless, some authors ${ }^{37}$ do not agree here.) Another important fact is that strangeness starts to grow in nuclear collisions at AGS energies, at which only hadronic matter is probably present. For example, $K^{+} / \pi^{+}$ratio increases from about .04 ( $p p$ or $p B e$ at such energies) to about .2 in $\mathrm{Si}$-Au collisions ${ }^{40}$.

Coming to theory, let us divide it into equilibrium and kinetic parts. Usual thermodynamical formulae can explain $K^{+}, K^{-}, \Lambda$ production at AGS with $T \sim 100 \mathrm{MeV}, n_{B}=.1$ $\mathrm{fm}^{-3}$ (see e.g. J. Cleymans talk in this proceedings). But can reactions in hadronic matter keep strangeness in equilibrium at such low temperatures (even lower than seen in $p_{t}$ spectra)? I think it cannot be the case, and here we certainly have non-equilibrium situation, even at much higher $T$ and should do kinetics. Unfortunately, in literature one can find quite different estimates. In particular, some people ${ }^{38}$ use large cross section $\sigma(\pi \pi \rightarrow K \bar{K})$ of the order of $3 \mathrm{mb}$, while in Ref. 39 it is argued, that standard one-meson exchange model gives for this cross section estimates about ten times smaller.

The main point of Ref. 39 is that production of strangeness is very sensitive to meson modification in matter. The simplest effect (in fact, not discussed in this paper, but considered above) is that attraction between pions (the modified dispersion curve) leads to essentially larger pion density at the same temperature. This by itself can increase the rate by an order of magnitude near $T_{c}$. The second effect is that in dense nuclear matter the kaon energy is expected to be shifted down. If so, it can enhance $K$ production rate, for it contains $\exp \left(-2 m_{K} / T\right)$. The shift in $m_{\rho}$ may make the process $\rho \rho \rightarrow K \bar{K}$ to be also very important ${ }^{39}$.

Let us now discuss the situation with strangeness in CERN experiments. Now we have a set of new data, with ratios like $\Xi / \Xi=0.43 \pm 0.07$ (WA85), $\bar{\Lambda} / \Lambda$ about $1 / 4, \Lambda / K_{\text {s }}$ about 2 (NA35), etc. They also can be fitted by equilibrium thermodynamics ${ }^{41}$, now at $T=200$ $\mathrm{MeV}$ and $\mu_{B}=180 \mathrm{MeV}, \mu_{s}=45 \mathrm{MeV}$. (Note, that here we have one parameter, not three: the first is taken from observed slope and two others are related by strangeness and baryon number conservations, so it is a nontrivial fit.) $I$ think at such $T$, just after QGP hadronization, one may probably believe in strangeness equilibration, but much more work is certainly needed to prove or disprove it.

It has been known for some time that in QGP strangeness is equilibrated soon enough, during few $\mathrm{fm} / \mathrm{c}$. One new idea, pointing in the same direction, was recently pointed out in Ref. 42. These authors argue that if gluons obtain large effective mass above $2 m$, one has 
decay of the gluon $g \rightarrow s \bar{s}$, which then makes strangeness equilibration time even several times shorter.

\section{DIRECT PHOTONS AND DILEPTONS}

Production of the "penetrating probes", direct photons and dileptons, was suggested as the best signal for QGP formation long ago ${ }^{29}$. The "window" for them to be seen was indicated to be at photon $p_{t}$ and/or dilepton invariant masses about $2-4 \mathrm{GeV}$.

WA80 has looked recently for direct photons up to $2 \mathrm{GeV} / \mathrm{c}$, but did not found them there. NA38 dilepton data about continuum are not yet presented in absolute normalization form, so they were not really analyzed theoretically. Thus much better data are needed in order to get reliable information about the hottest stages of the collisions.

\section{2. $J / \psi$ SUPPRESSION AND $\phi$ ENHANCEMENT}

NA38 dilepton experiment has found two interesting phenomena related to heavy vector mesons. $J / \psi$ production in central nuclear collisions is suppressed compared to $p p$ or peripheral ones, roughly by factor 2 , while production of $\phi$ meson is enhanced by factor 3 . In the first approximation, these opposite trends are consistent with the idea, that strangeness is thermally produced in matter, while charm is too heavy for that, and matter can only help to drag two $c$ quarks apart and destroy $J / \psi$.

However, as one wants to understand whether $J / \psi$ suppression is indeed due to charmed quark deconfinement in the plasma, as suggested originally in Ref. 43, a lot of questions arise. It can be due to $J / \psi$ splitting in hot hadronic matter ${ }^{45}$. In order to answer them one may use the idea suggested by Karsch and Petronzio" $J / \psi$ can jump out of the "hot spot" if its $p_{t}$ is large enough. And indeed, at $p_{t}>2 \mathrm{GeV}$ the observed suppression disappears. Taken literally, it means that the "suppression time" $\tau_{Q G P}$ only slightly exceeds the $J / \psi$ formation time $\tau_{\psi}$. This seems strange as $\tau_{\psi}$ is probably small, $1 / 2-1 \mathrm{fm} / \mathrm{c}$. If one assumes strong suppression at the hadronic stage as well, he obtains suppression at much larger $p_{T}$ (remember, size at breakup is about $8 \mathrm{fm}$ !), thus this last explanation seems to be rejected. Another possible reason for more $J / \psi$ at larger $p_{t}$ is "initial stage rescattering" of the gluons, producing the charmed quarks ${ }^{46}$. It got support from recent FNAL experiments (E772), in which similar suppression in $p A$ collisions were observed.

These issues can presumably be clarified by future experiments with $\mathrm{Pb}$ beams. In this case we certainly have larger $\tau_{\mathrm{QGP}}$, and if the Karsch-Petronzio idea is right, suppression should persist up to much higher $p_{t}$. If initial gluon rescattering or $\chi$ absorption is the case, we will see no big differences between $O^{16} A u$ and $\mathrm{Pb} A u$ collisions.

One more possibility (M. Strikman, private communication) is based on the observation that about $40 \%$ of $J / \psi$ are known to originate from $\chi$ decays. Can it be $\chi$, not $J / \psi$ suppression? Data on $\psi^{\prime}$ suppression (similar in size to $\chi$ ) can tell us more on that. 
Coming now to $\phi$ enhancement, we have to worn the reader, that what was said above about $\phi$ modification is not relevant for NA38 data, which correspond to high $p_{t}>1.3 \mathrm{GeV}$. If one adopts an "evaporation" scenario, he finds that the main effect here is probably the larger mean free path for $\phi$ relative to $\rho, \omega$ mesons, so that $\phi$ can be "evaporated" from inside and its enhancement may be just volume-to-surface effect. Again, direct check is to take the larger system using lead beam.
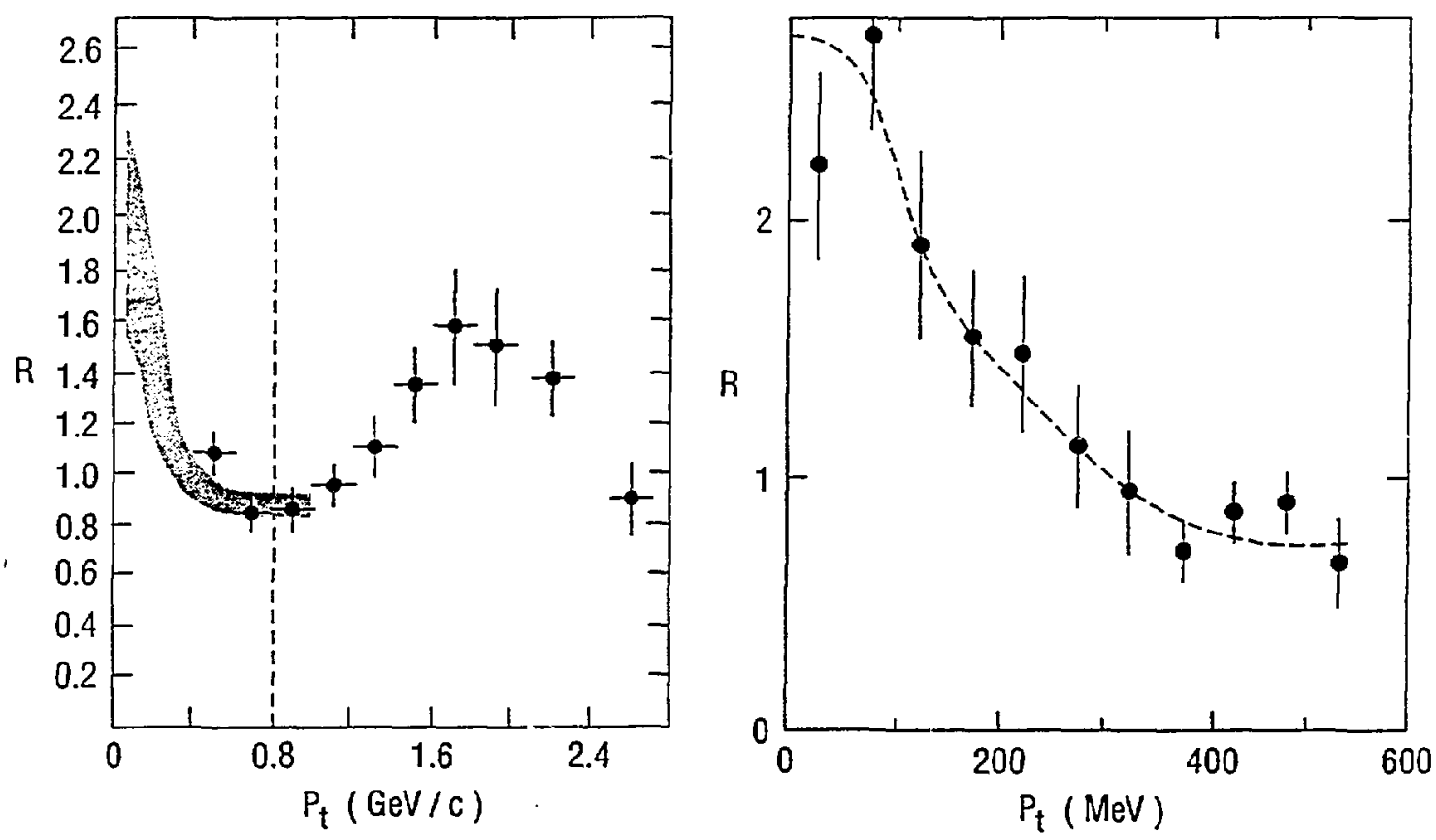

Figure 5: (a) The ratio of the $p_{t}$ spectra of $\pi^{0}$ in central and peripheral OW collisions (WA80) (points), while the shaded area is the ratio of $\pi^{-}$HELIOS spectra for SS collisions to pp ones. (b) The ratio of the spectra calculated in the quasipion model with and without modification of the dispersion curve.

\section{THE SOFT PION PUZZLE}

Now we return to "soft pion puzzle", or enhancement of soft pion production in central nuclear collisions compared to pp ones, see Fig. 5(a). It is so puzzling because, due to Goldstone theorem, soft pion interaction should be small for all types of processes. Therefore, in any kinetic calculations one might expect a dip at low momenta.

We have already criticized interpretation of soft pion peak by small $T$. Another suggested explanation, due to positive pion chemical potential ${ }^{48}$, claims to have kinetic explanation, but that was not explicitly demonstrated. Also, if one takes very big systems, in which 
equilibration in pion number should take place, then according to this idea the peak should disappear. One more explanation is the trace of resonance decays ${ }^{49}$, but this is essentially the same effect as discussed below. Different formulations depend on whether or not the system lifetime exceeds that of the resonances.

Pion modification plus boundary phenomena discussed above can provide a semi-quantitative explanation of the soft pion component at small $p_{t}$. Climbing out of the potential well, the outgoing pion reduces its momenta (see Fig. 2(b)). If the dispersion curve is flat at low momenta, large phase space corresponds to soft outgoing pions. Monte Carlo simulations ${ }^{35}$ have demonstrated very effective production of nearly stopping pions. Results of Fig. 5(b) roughly correspond to data. Another consequence of the collective attractive potential is that some pions are reflected from the boundary, which makes the system lifetime longer. This can be checked by interferometry, also it is very important for photon production we discuss in the next section.

\section{THE SOFT PHOTON PUZZLE}

The second striking phenomenon observed in high energy collisions during the last years is observation ${ }^{50}$ of an excess of the produced soft photons over the theoretical expectations. The reason this is disturbing is that emission of the soft photons is described by classical electrodynamics, which demands the simple bremsstrahlung to be the main effect, provided photon energies $\omega$ are small compared to inverse lifetime of the radiating system.

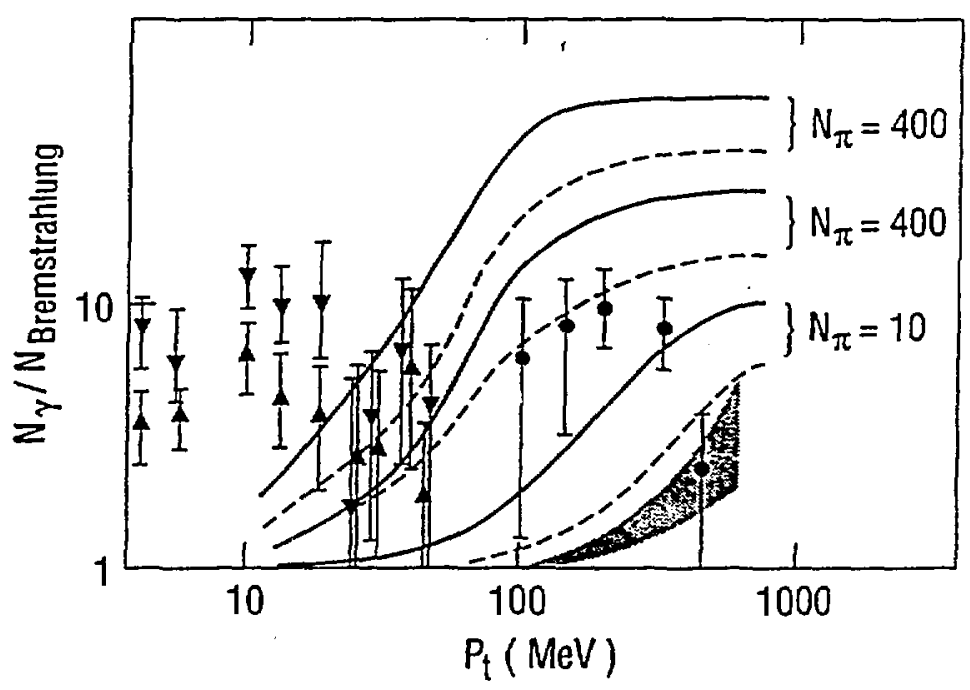

Figure 6: The ratio of the observed photon yield to bremsstrahlung predictions, versus $p_{t}$. The dashed area shows predictions of the Lund model $l^{53}$, while solid and dashed curves are for the quasipion model with and without pion modification ${ }^{35}$. 
Attempts to describe these data in conventional models of the collisions has failed, (see the shaded region in Fig. 6). Soft photon production was also evaluated in the "quasipion" model in Ref. 35. The results shown in Fig. 6 show significant deviations from bremsstrahlung predictions. The main reason for that is that pions are rescattered several times before they come out of the system.

Comparing results of the "quasipion" cascades with data, one may conclude that they definitely cannot describe the observed excess for $p_{t}<30 \mathrm{MeV}$. However, they do predict significant enhancement over bremsstrahlung at larger $p_{t}$, and account for pion modification increases it roughly by the factor of two (more in the transition region, between coherent and incoherent regimes of radiation). Note also, that the data shown by solid points in Fig. 6 comes from dileptons, and they have smaller background. These data (inside their big errors) are consistent with our predictions. Finally, for very large multiplicity ( $\mathrm{Pb} \mathrm{Pb}$ collisions) the model predicts very strong photon enhancement, which is a direct measure of rescatterings. Its experimental check is of great interest.

\section{OTHER "ULTRASOFT" PHENOMENA}

Recently first dilepton experiments at Berkeley ${ }^{51}$ have studied the lepton pair production at energies as low as $4.9 \mathrm{GeV}$ for proton-nucleus and about $1 \mathrm{GeV} / \mathrm{N}$ in $\mathrm{Ca} \mathrm{Ca}$ collisions. The data are well described by known sources, except for the peak just above double pion mass. One may think about $\pi^{+} \pi^{-}$annihilation, and in fact $s$-dependence of this peak is consistent with that for pion pair production. However, attempts to ascribe it to pion annihilation meet certain problems. First of all, production of two soft pions is suppressed by smallness of the phase space. Second, the annihilation of soft pions into a virtual photon is suppressed at threshold because it takes place in the $P$ wave.

Let us emphasize that conditions of this experiment are quite different from nuclear collisions at CERN. There are no hundreds of pions in a fireball, but just few. However, in this case pions are produced and annihilated inside the nuclear matter. Gale and Kapusta ${ }^{52}$ have suggested, that pion dispersion curve is modified in compressed nuclear matter, so that secondary minimum in $\omega(k)$ is developed. If so, annihilation of two "quasipions" leads to a peak in the dilepton spectrum, which may well be mixed with the double-pion threshold. In such case there is no suppression mentioned above: in this scenario quasipion momentum is not small.

Let me make two comments here. Actually the suggested secondary minimum ${ }^{52}$ is not needed: instead, it is better if $\omega(k)$ has flat region at small $k$. The second one deals with the fact that naive estimates of the annihilation rate $^{52}$ give annihilation rate one order of magnitude less than observed. Pion motion was considered as that of a free classical particle, while it is a quantum particle moving in collective attractive potential, which may increase the annihilation probability.

The second unexplained phenomenon is strong clustering of pions, resulting in enhanced probability of events with "spikes" in rapidity distribution. The most famous one is the NA22 
event, in which 10 pions are in one bin with the width 0.1 , which certainly is not random fluctuation. The pions have somewhat smaller $p_{t}$ in it, but their $p_{l}$ range is only $20 \mathrm{MeV}$ or so in the "spike" CM system! Events like that does dominate higher moments, related to "intermittency" by some authors (see Bialas talk in this Proceedings and references therein). I do not think analogy to turbulence etc. can help much. If the effect is real, its explanation is probably due to some specific "final state interaction". Van Hove ${ }^{54}$ suggested it to be metastable droplets of QGP, but it may also be due to collective attractive interaction of several pions.

\section{REFERENCES}

1. E.V. Shuryak, Phys. Lett. 78B (1978) 150, Yad. Fis. 28 (1978) 796. The QCD Vacuum, Hadrons and the Superdense Matter, World Scientific 1988;

D.J. Gross, R.D. Pisarsky and L.G. Yaffe, Rev. Mod. Phys. 53 (1981) 43;

B. Muller, Lecture Notes in Physics 225, (Springer-Verlag 1985);

J. Cleymans, R.V. Gavai and E. Suchonen. Phys. Rep. C130 (1986) 217.

2. Proceedings of Quark Matter ' 88 , Nordkirhen, Z. Phys. C. 38 (1988);

Proceedings of Quark Matter '89, Lenox, Nucl. Phys. A498 (1989);

Proceedings of Quark Matter '90, , Nucl. Phys. (in press)..

3. E.V. Shuryak, Phys. Lett. B107 (1982)103.

4. P. Bacilieri et al., (APE coll.) Phys.Lett.B220 (1989) 607.

5. J.B. Kogut et al., Phys. Rev. D39 (1989) 636 S. Gottlieb et al., Phys. Rev. D40 (1989) 2389 M.P. Crady et al., Nucl. Phys. B200 (1988) 149.

6. N.H. Crist, Columbia preprint CU-TP-447;

S. Ohta, Columbia preprint CU-tp-446.

7. M. Gell-Mann and M. Levy, Nuovo Cimento 16 (1960) 705.

8. J. Gasser and H. Leutwyler, Phys. Rep. 87 (1982) 79.

9. G. 't Hooft, Phys. Rev. D14 (1976) 3432.

10. E.V. Shuryak, Nucl. Phys. B203 (1982) 93,116,140.

11. D.I. Dyakonov and V. Yu. Petrov, Nucl. Phys. B245 (1984) 259, B272 (1986) 457.

12. E.M. Ilgenfritz and E.V.Shuryak, Nucl. Phys. B319 (1989) 511.

13. E.V. Shuryak, Nucl. Phys. B302 (1988) $559,574,599,621$.

14. E.V. Shuryak and J.J.M. Verbaarschot, CERN-TH-5492/89, Nucl. Phys. in press..

15. E.V. Shuryak, Nucl. Phys. B319 (1989) 521,541; B328 (1989) 85,102.

16. R. Baier, B. Pire and D. Schiff, Phys. Rev. 38D (1988) 2814;

T. Altherr, P. Aurenche and T. Becherrawy, Nucl. Phys. B315 (1989) 436;

A. Mahlin, Dilepton spectrum from QGP in second Born approximation, ITP-89-72E, Kiev 1989.

17. E. Braaten and R.D. Pisarski, BNL-43390, Nucl. Phys. in press.

18. J. Frenkel and J.C. Taylor, Cambrige Univ. DAMTP-89-23, Nucl. Phys., in press.

19. E. Braaten, R.D. Pisarski, T.C. Yuan, Production of soft dileptons in quark-gluon plasma, BNL-43882, NUHEP-th-90-1, 1990.

20. H.A. Weldon, Measuring $T_{c}$ of the quark-gluon plasma with $\epsilon^{+} e^{-}$pairs, West Virginia University preprint, 1990.

21. P. Gerber and H. Leutwyler, Bern Univ. preprint BUTP-SS/30 (Nucl.Plyss., in press). 
22. E.V. Shuryak, Jad. Fis. (Sov. J. of Nucl. Phys.) 16 (1972) 395.

23. G.M. Welke, R. Venugopalan and M. Prakash, The speed of sound in an interacting pion gas, SUNY-NTG-90-20.

24. E.V. Shuryak, Phys. Lett. 207B (1988) 345.

25. J.L. Goily and H. Leutwyler, Bern University Preprint 1990.

26. G.E. Brown, Matter under extreme conditions, SUNY-NTG-90-13.

27. E.V. Shuryak, Yadernaya Fisika (Sov. J. of Nucl. Phys.) 16 (1972) 395.

28. A. Bialas, Talk at Quark Matter '88, Lennox..

29. E.V. Shuryak, Yadernaya Fisika (Sov. J. of Nucl. Phys.) 16 (1972) 395.

30. E.V. Shuryak and O.V. Zhirov, Phys. Lett. 89B (1980) 253.

31. M. Kataja, P.V. Ruuskanen, L.D. McLerran and H. von Gersdorff, Phys. Rev. D34 (1986) 2755.

32. T. Alexopoulos et al., Phys. Rev. Lett. 64 (1990) 991;

F. Turkot, Talk at Quark Matter '90, Menton, 1990.

33. G. Odyniec (NA35), Talk at Workshop on Relativistic Aspects of Nuclear Physics, Rio de Janeiro, 1989 (WSPC, Singapore, in press).

34. K.S. Lee, U. Heinz and E. Schnedermann, Search for collective... preprint TPR-90-18, Regensburg, submitted to Zeit. Phys. C.

35. E.V. Shuryak, Pion modification in hot hadronic matter and "ultrasoft" phenomena in high energy collisions, CERN-TH-5386/89 and "L. Van Hove Festschrift", World Scientific, (1990). Physics of the pion liquid, BNL-44522, 1990.

36. K. Miyano et al., Phys. Rev. Lett. 53 (1984) 1725 and later preprints.

37. J. Rafelski, Phys. Lett. 207B (1988) 371.

38. J. Kapusta and A. Mekjian, Phys. Rev. C33 (1986) 1304.

39. C.M. Ko, Z. Wu, L.H. Xia, G.E. Brown, Proceedings of the HIPAGS Workshop, BNL, March 1990.

40. Y .Miake, Talk at HIPAGS Workshop, BNL, March 1990.

41. C.B. Dover, Theoretical Overview of RHIC Physics, talk at Annual Meeting of the AGS users group, BNL-44621, May 1990.

42. T.S. Biro, P. Levai and B. Muller, Strangeness production with "massive" gluons, Durham, NC, 1990.

43. T. Matsui and H. Satz, Phys. Lett. 178b (1986) 416.

44. F. Karsch and R. Petronzio, Z. Phys. C37 (1988) 627.

45. J. Ftacnik, P. Lichard and J. Pisut, Phys. Lett. B207 (1988) 194;

S. Gavin, M. Gyulassy and A. Jackson, Phys. Lett. 207B (1988) 257.

46. S. Gavin and M. Gyulassy, Phys. Lett. 214B (1988) 241;

J. Hufner, Y. Kurihara and H.J. Pirner, Phys. Lett. 215B (1988) 218;

J.P. Blaizot and J.Y. Ollitrault, Phys. Lett. 217B (1989) 392.

47. C. Baglin et al., (NA38 coll.) Phys. Lett. 220B (1989) 471;

J. Varela, Talk at Quark Matter '90, Menton..

48. M. Kataja and P.V. Ruuskanen, University of Jyvaskyla preprint 1/90, January 1990.

49. G.E. Brown, J. Stachel and G.M. Welke, Hot and Cool Baryons and Cool Pions in Relativistic Heavy Ion Reactions, preprint of SUNY, Stony Brook 1990.

50. P.V. Chliapnikov et al., Phys. Lett. 141B (1984) 276; 
U. Gurlach, (HELIOS coll.) Proc. of 24-th Int. Conf. High. Energy Phys., Munich 1988;

W.J. Willis, Nucl. Phys. A478 (1988) 151C;

J. Schukraft, (HELIOS coll.) Talk at 7th Int. Conf. Quark Matter '88, Lenox, Massachusetts 1988.

51. G. Roche et al., (DSL coll.) First observation of dielectron production at the Bevalac, LBL preprint, Berkeley 1988;

L.S. Schroeder. Talk at HIPAGS Workshop, BNL, March 1990.

52. C. Gale and J. Kapusta, Phys. Rev. C35 (1987) 2107;

L.H. Xia et al., Nucl. Phys. A485 (1988) 721;

J. Kapusta, Talk at HIPAGS Workshop, BNL, March 1990.

53. B. Andersson et al., Preprint LUTP 88-1, Lund 1988.

54. L. Van Hove, CERN preprint TH 5236/88, 1988.

55. H. Leutwyler and A. Smilga, BERN University preprint, 1990.

56. L. Van Hove, Z. Phys. C21 (1983) 93.

\section{DISCLAIMER}

This report was prepared as an account of work sponsored by an agency of the United States Government. Neither the United States Government nor any agency thereof, nor any of thejr employees, makes any warranty, express or implied, or assumes any legal liability or responsibility for the accuracy, completeness, or usefulness of any information, apparatus, product, or process disclosed, or represents that its use would not infringe privately owned rights. Reference herein to any specific commercial product, process, or service by trade name, trademark, manufacturer, or otherwise does not necessarily constitute or imply its endorsement, recommendation, or favoring by the United States Government or any agency thereof. The views and opinions of authors expressed herein do not necessarily state or reflect those of the United States Government or any agency thereof. 